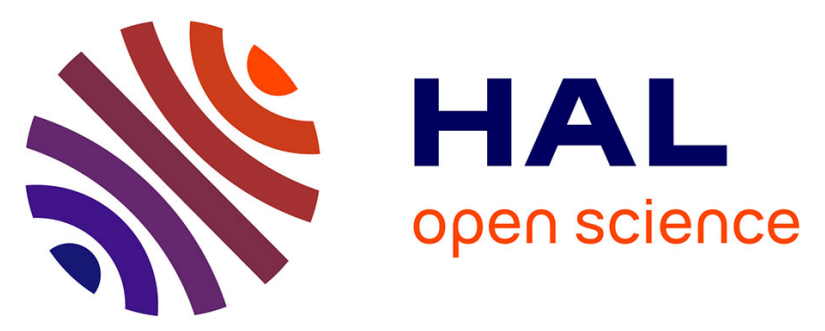

\title{
The murine Muc2 mucin gene is transcriptionally regulated by the zinc-finger GATA-4 transcription factor in intestinal cells
}

\author{
Maria van Der Sluis, Monique H M Melis, Nicolas Jonckheere, Marie-Paule \\ Ducourouble, Hans A Bü Ller A, Ingrid Renes, Alexandra Einerhand, Isabelle \\ van Seuningen
}

\section{To cite this version:}

Maria van Der Sluis, Monique H M Melis, Nicolas Jonckheere, Marie-Paule Ducourouble, Hans A Bü Ller A, et al.. The murine Muc2 mucin gene is transcriptionally regulated by the zinc-finger GATA-4 transcription factor in intestinal cells. Biochemical and Biophysical Research Communications, 2004, 325, pp.952 - 960. 10.1016/j.bbrc.2004.10.108 . hal-02905675

\section{HAL Id: hal-02905675 \\ https://hal.science/hal-02905675}

Submitted on 29 Sep 2020

HAL is a multi-disciplinary open access archive for the deposit and dissemination of scientific research documents, whether they are published or not. The documents may come from teaching and research institutions in France or abroad, or from public or private research centers.
L'archive ouverte pluridisciplinaire HAL, est destinée au dépôt et à la diffusion de documents scientifiques de niveau recherche, publiés ou non, émanant des établissements d'enseignement et de recherche français ou étrangers, des laboratoires publics ou privés. 


\title{
The murine Muc2 mucin gene is transcriptionally regulated by the zinc-finger GATA-4 transcription factor in intestinal cells ${ }^{\text {is }}$
}

\author{
Maria van der Sluis ${ }^{\mathrm{a}, \mathrm{b}}$, Monique H.M. Melis ${ }^{\mathrm{a}}$, Nicolas Jonckheere ${ }^{\mathrm{c}}$, \\ Marie-Paule Ducourouble ${ }^{\mathfrak{c}}$, Hans A. Büller ${ }^{\mathrm{a}}$, Ingrid Renes ${ }^{\mathrm{b}}$, \\ Alexandra W.C. Einerhand ${ }^{\mathrm{a}}$, Isabelle Van Seuningen ${ }^{\mathrm{c}, *}$

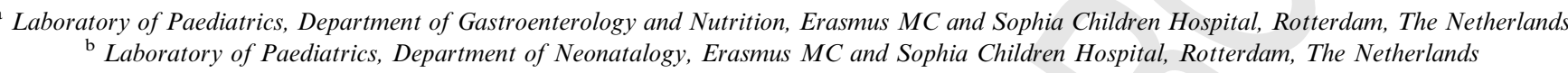

Received 14 October 2004

\section{Abstract}

MUC2, the major mucin in the intestine, is expressed early during development and shows an altered expression pattern in intestinal bowel diseases. However, the mechanisms responsible for MUC2 expression in the intestine during these events are largely unknown. Having found putative GATA binding sites in the murine Muc2 promoter and that GATA-4 is expressed in Muc2-expressing goblet cells of the mouse small intestine, we undertook to study its regulation by this transcription factor. A panel of deletion mutants made in pGL3 vector and covering $2.2 \mathrm{~kb}$ of the promoter were used to transfect the murine CMT-93 colorectal cancer cell line. The role of GATA-4 on Muc2 gene regulation was investigated by RT-PCR and co-transfections in the presence of expression vectors encoding either wild-type or mutated GATA-4 or by mutating the GATA-4 site identified within Muc2 promoter. Four GATA-4 cis-elements were identified in the promoter by EMSA and Muc2 promoter was efficiently activated when GATA-4 was overexpressed in the cells with a loss of transactivation when those sites were either mutated or a mutated form of GATA-4 was used. Altogether, these results identify $\mathbf{M u c 2}$, a goblet cell marker, as a new target gene of GATA-4 and point out an important role for this factor in Muc2 expression in the intestine.

(c) 2004 Elsevier Inc. All rights reserved.

Keywords: Muc2; Mucin; GATA-4; Intestine; Goblet cell; Differentiation; Transcription

The mucus layer in the lumen of the gut forms a physical barrier against microorganisms and insoluble material [1]. Goblet cells are a hallmark of the intestinal epithelium, and are known to secrete mucins that participate in mucus formation to protect the underlying mucosa. The secretory mucin Muc2, a very large $O$-glycoprotein, is particularly relevant for the study of goblet

\footnotetext{
Abbreviations: bp, basepair; EMSA, electrophoretic mobility shift assay; kb, kilobase; LPH, lactase phlorizin hydrolase; SI, sucrase isomaltase.

${ }^{*}$ Corresponding author. Fax: +33 320538562 .

E-mail address: isabelvs@lille.inserm.fr (I. Van Seuningen).
}

cell biology as it is exclusively and abundantly expressed by intestinal goblet cells $[2,3]$. It is produced throughout the gastro-intestinal tract increasing from small intestine to colon.

Muc2 is expressed early during embryonic development of the intestine [4], which suggests that $M u c 2$ transcription is under the influence of transcription factors responsible for intestinal development and cell differentiation. The early expression of mucin before mucus cell differentiation or during the process of differentiation indicates that they may be the targets of transcription factors responsible for those programs [5]. This hypothesis has been recently confirmed in a previous study 
47 where we showed that the human $M U C 2$ mucin gene is 48 regulated by homeobox $\mathrm{Cdx} 1$ and $\mathrm{Cdx} 2$ transcription 49 factors [6], which are both involved in intestinal cell dif50 ferentiation [7].

GATA transcription factors belong to another fam-

52 ily of transcription factors involved in development

53 and cell differentiation. GATA factors are zinc fingers

54 and are classified into two subfamilies based on struc-

55 tural features and expression patterns. GATA-1,

56 GATA-2, and GATA-3 are involved in hematopoiesis

57 and neurogenesis whereas GATA-4, GATA-5, and

58 GATA-6 possess overlapping patterns in the develop-

59 ing cardiovascular system and in endoderm-derived

60 tissues including the liver, lungs, pancreas, stomach,

61 and intestine [8].

62 During embryonic development GATA-4 mRNA is

63 expressed in the primitive intestine $[9,10]$ in which

$64 M u c 2$ is also found [4]. Its expression in the intestine is

65 sustained during adulthood with a distinct expression

66 pattern along the crypt-villus axis, which strongly corre-

67 lates with the status of differentiation of the cells. It is

68 highly expressed on the tips of the villi and its expression

69 decreases as we go downward towards the crypt [11].

70 Moreover, GATA factors are known to regulate intes-

71 tine-specific genes, which are considered as markers of

72 enterocytes, such as fatty acid binding protein [11],

73 intestinal lactase-phlorizin hydrolase (LPH) [11-13],

74 and sucrase isomaltase (SI) [14]. However, no GATA-

754 target gene, representative of goblet cells, which are

76 responsible for mucus production/secretion and mainte-

77 nance of a defense line and protective barrier in intesti-

78 nal pathophysiology [1], has been identified so far.

79 Characterization of the 5'-regulatory regions of

80 numerous genes has demonstrated that GATA factors

81 interact with a DNA sequence element containing the

82 core GATA motif (A/T)GATA(A/G) [8]. Computer

83 analysis of the murine $M u c 2$ promoter sequence [15] re-

84 vealed the presence of several putative GATA binding

85 sites throughout the promoter region.

86 Altogether, these data are in favor of a role for

87 GATA-4 in regulating $M u c 2$ gene expression. Having

88 found binding sites for that factor in the promoter of

$89 M u c 2$, and because of the spatio-temporal restricted pat-

90 tern of expression of MUC2 and GATA-4 along the

91 crypt-villus axis in the intestine, we undertook to study

92 the regulation of the promoter of mouse $M u c 2$ by

93 GATA-4 in a murine colorectal cancer cell line. Implica-

94 tions for MUC2 expression during intestine develop-

95 ment and cell differentiation are discussed.

\section{Materials and methods}

97 Animals. Adult specified pathogen-free Balb/c mice, obtained from

98 Harlan (Zoetermeer, The Netherlands), were killed by cervical dislo-

99 cation. The intestine was removed, fixed in $4 \%$ paraformaldehyde in
PBS, and subsequently processed for light microscopy as described previously [16]. The animal experiments were performed with the approval of the Animal Studies Ethics Committee of the Erasmus MC (Rotterdam, The Netherlands)

Immunohistochemistry. Five micrometers of thick paraffin sections were cut and deparaffinized through a graded series of xylol-ethanol. Endogenous peroxidase activity was inactivated by $1.5 \%$ (v/v) hydrogen peroxide in PBS for $30 \mathrm{~min}$, followed by antigen retrieval in $0.01 \mathrm{M}$ citrate buffer for $10 \mathrm{~min}$ at $100^{\circ} \mathrm{C}$. Thereafter, sections were incubated with TENG-T (10 mM Tris-HCl, $5 \mathrm{mM}$ EDTA, $150 \mathrm{mM} \mathrm{NaCl}, 0.25 \%$ $(\mathrm{w} / \mathrm{v})$ gelatin, and $0.05 \%(\mathrm{w} / \mathrm{v})$ Tween 20 ) for $30 \mathrm{~min}$ to reduce nonspecific binding. This was followed by overnight incubation with a 1:2500 dilution of goat anti-GATA-4 antibody (sc-1237X, Santa Cruz Biotechnology, USA) in PBS containing $1 \%(\mathrm{w} / \mathrm{v})$ bovine serum albumin and $0.1 \%(\mathrm{v} / \mathrm{v})$ Triton $\mathrm{X}-100$. Then, the sections were incubated for $1 \mathrm{~h}$ with biotinylated horse anti-goat IgG (diluted 1:2000, Vector Laboratories, England) followed by a $1 \mathrm{~h}$ incubation with ABC/PO complex (Vectastain Elite Kit, Vector Laboratories) diluted $1: 400$. Binding was visualized after incubation in $0.5 \mathrm{mg} / \mathrm{ml}$ of $3,3^{\prime}$ diaminobenzidine (DAB), $0.02 \%(\mathrm{v} / \mathrm{v}) \mathrm{H}_{2} \mathrm{O}_{2}$ in $30 \mathrm{mM}$ imidazole, and $1 \mathrm{mM}$ EDTA ( $\mathrm{pH}$ 7.0). To visualize goblet cells, sections were stained with Alcian Blue 8GX (BDH, Brunschwig Chemie, Amsterdam, The Netherlands). Finally, sections were dehydrated and mounted.

Muc2-pGL3 deletion mutant constructs. The Muc2-pGL3 deletion mutants that cover $2.2 \mathrm{~kb}$ of the promoter (GenBank Accession No. AF221746) were constructed into promoterless pGL3 Basic vector (Promega) using a PCR-based method as described previously $[17,18]$. PCRs were carried out on mouse genomic DNA using the primers depicted in Table 1. PCR products were then subcloned into pCR2.1 vector (Invitrogen) before subcloning into $B g / \mathrm{II}-M l u \mathrm{I}$ sites of the pGL3 Basic vector previously linearized with the same restriction enzymes. All clones were sequenced on both strands on an automatic LICOR sequencer using infrared-labeled RV3 and GL2 primers (Promega). Plasmids used for transfection studies were prepared using the Endofree plasmid Mega kit (Qiagen).

Cell culture. Murine rectal cancer cell line CMT-93 was cultured as described in [19]. IEC-6 cells were purchased from the European Collection of Animal Cell Cultures (ECACC). This cell line was established from rat small intestine crypt cells and was cultured in Dulbecco's modified essential medium containing $5 \%$ fetal bovine serum, $2 \mathrm{mM}$ L-glutamine, $10 \mu \mathrm{g} / \mathrm{ml}$ insulin, $50 \mathrm{U} / \mathrm{ml}$ penicillin, and $50 \mu \mathrm{g} / \mathrm{ml}$ streptomycin. All cells were cultured at $37^{\circ} \mathrm{C}$ in a humidified $5 \% \mathrm{CO}_{2}$ water-jacketed incubator. All reagents were from Invitrogen (Gibco) unless otherwise indicated.

Transfections. Transfection and co-transfection experiments were performed using Effectene reagent (Qiagen) as described previously [18] using $1 \mu \mathrm{g}$ Muc2-pGL3 deletion mutants. Total cell extracts were prepared after a $48 \mathrm{~h}$ incubation at $37^{\circ} \mathrm{C}$ using $1 \times$ Reagent Lysis Buffer (Promega) as described in the manufacturer's instruction manual. Luciferase activity $(20 \mu \mathrm{l})$ was measured on a TD 20/20 luminometer (Turner Design). Total protein content in the extract $(4 \mu \mathrm{l})$ was measured using the bicinchoninic acid method in 96-well plates as described in manufacturer's instruction manual (Pierce, Bezons, France). In co-transfection experiments, $1 \mu \mathrm{g}$ of the deletion mutant of interest was transfected with $0.25 \mu \mathrm{g}$ of the expression plasmid encoding the transcription factor of interest (wild-type pcDNA-(WT) GATA-4 and mutated forms pcDNA-(-Act) GATA-4 and pcDNA-(C290S) GATA-4) [13]. Results were expressed as fold activation of luciferase activity in samples co-transfected with the transcription factor of interest compared with the control co-transfected with the corresponding empty vector. To study the effect on endogenous $M u c 2$ expression, cells $\left(0.5 \times 10^{6}\right)$ were transfected with $4 \mu \mathrm{g}$ of the expression vector of interest, and cultured for $48 \mathrm{~h}$ before being lysed and processed for total RNA preparation.

$R T-P C R$. Total RNA from CMT-93 cells was prepared using the RNeasy mini-kit from Qiagen. $1.5 \mu \mathrm{g}$ of total RNA was used to prepare cDNA (Advantage RT-for-PCR kit, Clontech). PCR was
100

101

102

103

104

105

106

107

108

109

110

111

112

113

114

115

116

117

118

119

120

121

122

123

124

125

126

127

128

129

130

131

132

133

134

135

136

137

138

139

140

141

142

143

144

145

146

147

148

149

150

151

152

153

154

155

156

157

158

159

160

161

162

163

164

165

166 
Table 1

Sequences of the pairs of primers used in PCR to produce deletion mutants covering the $M u c 2$ promoter

\begin{tabular}{lll}
\hline Position in the promoter & Oligonucleotide sequence $\left(5^{\prime} \rightarrow 3^{\prime}\right)$ & Orientation \\
\hline$-221 /+29$ & CGCACGCGTTTGGGGCTATGACATCCTGA & Sense \\
& CGCAGATCTGGTGGCTCACGAGGGTGGCAC & Antisense \\
$-563 /+29$ & CGCACGCGTATGGGGTCAGACACCCGT & Sense \\
& CGCAGATCTGGTGGCTCACGAGGGTGGCAC & Antisense \\
$-729 /+29$ & CGCACGCGTGAGGGCTGCCCAAGTTTAA & Sense \\
& CGCAGATCTGGTGGCTCACGAGGGTGGCAC & Antisense \\
$-1001 /+29$ & CGCACGCGTGGCAAGCCCAGGGACTGAAG & Sense \\
& CGCAGATCTGGTGGCTCACGAGGGTGGCAC & Antisense \\
$-1568 /+29$ & CGCACGCGTGAGGTGGGAGGACTGGCTTC & Sense \\
$-2213 /+29$ & CGCAGATCTGGTGGCTCACGAGGGTGGCAC & Antisense \\
& CGCACGCGTTCTTGGTCCTCAACCAAAGTT & Sense \\
& CGCAGATCTGGTGGCTCACGAGGGTGGCAC & Antisense
\end{tabular}

BglII (AGATCT) and MluI (ACGCGT) sites (bold) were added at the end of the primers to direct subcloning into the pGL3 Basic vector.

performed on $5 \mu \mathrm{l}$ cDNA using a specific pair of primers (MWGBiotech, Germany) for mouse Muc2 mucin gene (forward primer: $5^{\prime}$ TGTGGCCTGTGTGGGAACTTT-3'; reverse primer: 5'-CATA GAGGGCCTGTCCTCAGG-3'). Mouse $\beta$-actin (forward primer: $5^{\prime}$ TCACGCCATCCTGCGTCTGGACT-3'; reverse primer: $5^{\prime}$-CCG GACTCATCGTACTCCT-3') was used as the internal control. PCRs were carried out in $50 \mu \mathrm{l}$ final solutions as previously described [6]. Annealing temperature was 62 and $59^{\circ} \mathrm{C}$, respectively. PCR products were analyzed on a $1.5 \%$ agarose gel run in $1 \times$ Tris-borate-EDTA buffer. One hundred base pair DNA ladder was purchased from Amersham Biosciences. Expected sizes for Muc2 and $\beta$-actin PCR products are 558 and $582 \mathrm{bp}$, respectively. RT-PCRs were carried out on cDNAs from three different sets of experiments.

Nuclear extract preparation. Nuclear extracts from CMT-93 cells were prepared as described by Van Seuningen et al. [20] and kept at $-80^{\circ} \mathrm{C}$ until use. Protein content ( $2 \mu \mathrm{l}$ of cell extracts) was measured using the bicinchoninic acid method as described above.

Oligonucleotides and DNA probes. Oligonucleotides used as probes and competitors in EMSAs are shown in Table 2. They were synthesized by MWG-Biotech (Ebersberg, Germany). Equimolar amounts of single-stranded oligonucleotides were annealed and radiolabeled using T4 polynucleotide kinase (Promega) and $\left[\gamma_{-}^{-32} \mathrm{P}\right] \mathrm{dATP}$. Radiolabeled probes were purified by chromatography on a Bio-Gel P-6 column (Bio-Rad).

Electrophoretic mobility shift assay. The sequence of Muc2 promoter was analyzed with MatInspector V2.2 and Alibaba2 software based on the genomatix and gene-regulation databases, respectively, to determine the location of putative transcription factor binding sites
[21]. Electrophoretic mobility shift assay (EMSA) were performed as described previously by Mesquita et al. [6]. Supershift analyses were carried out using $1 \mu \mathrm{l}$ of the anti-GATA- 4 antibody (Santa Cruz, sc1237X). Reactions were stopped by adding $2 \mu$ loading buffer. Samples were loaded onto a $4 \%$ non-denaturing polyacrylamide gel and electrophoresis conditions were as described in [18]. Gels were vacuumdried and autoradiographed overnight at $-80^{\circ} \mathrm{C}$.

Site-directed mutagenesis. QuickChange site-directed mutagenesis kit (Stratagene) was used to generate site-specific mutations in the two proximal GATA sites found in the Muc2 promoter at $-168 /-165$ and $-158 /-155$, respectively. The oligonucleotide containing the double mutation was designed according to manufacturer's instructions and its sequence is depicted in Table 2 .

\section{Results and discussion}

The GATA-4 transcriptions factor is expressed in mouse embryonic intestinal tissue as early as ED 9.5 [8]. Its importance in regulating several promoters of enterocyte-specific genes has been previously described [11-13]. The MUC2 mucin is a marker of another intestinal cell type, the goblet cell, and possesses a spatiotemporal pattern of expression in embryonic, fetal, and adult intestine [4,22]. However, regulation of MUC2 mucin expression during development is largely

Table 2

Sequences of the sense oligonucleotides used for EMSAs and for site-directed mutagenesis

\begin{tabular}{ll}
\hline & Sequence $\left(5^{\prime} \rightarrow 3^{\prime}\right)$ \\
\hline $\begin{array}{l}\text { Oligonucleotides used for EMSA } \\
\text { Wild-type T216 }(-99 /-96)\end{array}$ & \\
Mutated T216 & CACAGCTGTTTTCCTGATAACTTGGCA \\
Wild-type T168 $(-168 /-165,-158 /-155)$ & CACAGCTGTTTTCCTCTTAACTTGGCA \\
Mutated T168 & TCATATAAAGATAAACTCAGATAACCTG \\
Wild-type T225 $(-401 /-398)$ & TCATATAAACTTAAACTCACTTAACCTG \\
Wild-type T169 $(-1521 /-1518)$ & TCCTTATTCTATCTAGGCTGGGCT \\
Mutated T169 & TTGATCATTTTTATCTCTGATGTCTTT \\
\end{tabular}

Oligonucleotide used for site-directed mutagenesis Double mutant $(-168 /-165 ;-158 /-155)$

CCAGGGAGTCATATAAACTTAAACTCACTTAACCTGAATCA

Positions of the GATA binding sites, within the probe, relative to the Muc2 transcription start site are indicated in parentheses. Putative GATA binding sites in the sequence are bold and in italics. Putative HNF binding site in T216 at -108/-104 is in italics. Mutated nucleotides are bold and underlined. Antisense oligonucleotides were also synthesized and annealed to the sense oligonucleotides to produce double-stranded DNA. 
218 unknown and is mandatory to better understand intes219 tine differentiation and cell lineage establishment [23]. 220 In this work we studied the transcriptional regulation 221 of murine $M u c 2$ by GATA- 4 and mapped the cognate 222 cis-elements within the promoter. We focused on the 223 role of GATA-4 factor since it is known to play an 224 important role in directing cell lineage-specific gene 225 expression during development of the vertebrate gut.

226 GATA-4 is expressed in goblet cells of the mouse small 227 intestine

228 Muc2 is the main mucin expressed by intestinal gob229 let cells [2]. Alcian blue staining of the mouse small 230 intestine indicates the presence and location of these 231 goblet cells (Fig. 1). Alcian blue-positive goblet cells 232 are present both along the villi and in the crypts. Immu233 nohistochemical staining of the same section with a 234 GATA-4-specific antibody showed staining of nuclei of 235 all the cells along the crypt-villus axis, including goblet 236 cells (Fig. 1). We can thus conclude that GATA-4 tran-

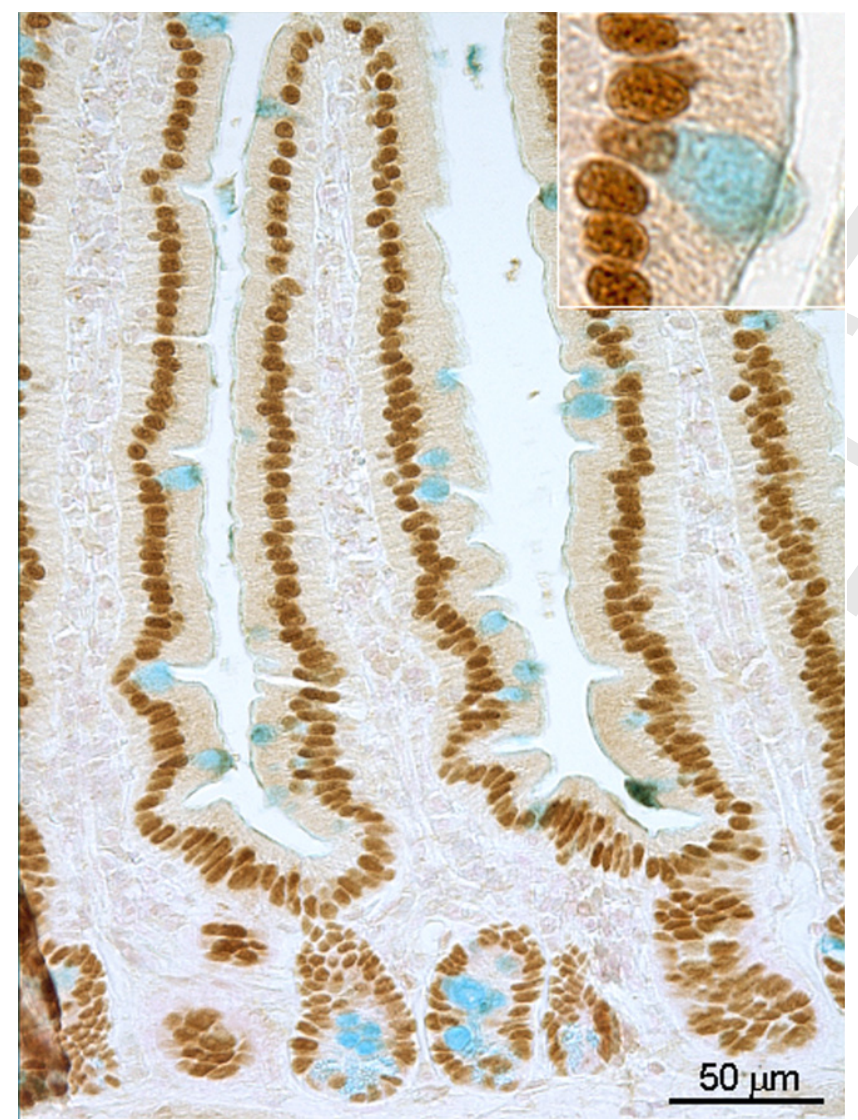

Fig. 1. Expression of GATA-4 transcription factor in mouse small intestine by immunohistochemistry. Alcian blue staining was performed as described in Material and methods. GATA-4 immunostaining was carried out on the same section of mid jejunum of an adult mice using a specific antibody for GATA-4. Sections were counterstained with hematoxylin. Inset: close-up of one GATA-4 expressing goblet cell. scription factor is expressed in Muc2-expressing goblet cells in the small intestine. The fact that GATA-4 has been shown to play an important role in the regulation of genes expressed in the intestine [11-13] and its co-localization with Muc2 in the goblet cells suggests an important role for GATA-4 in the regulation of $M u c 2$ gene. In order to confirm this hypothesis we first identified GATA-4 responsive regions within $M u c 2$ promoter by co-transfection experiments in the presence of wildtype or mutated forms of either Muc2 promoter or GATA-4 factor and then identified GATA-4 cis-elements by gel-shift assays.

Muc2 promoter activity in murine and rat intestinal cells

To define essential regions that drive $M u c 2$ transcription, six deletion mutants covering $2.2 \mathrm{~kb}$ of the promoter were constructed in the promoterless pGL3 Basic vector (Fig. 2A). Numbering refers to the transcription start site designated as +1 that was previously described [24]. These constructs were then transfected into two different cell lines, a goblet-like cell line CMT-93 [25] and a crypt-like cell line IEC-6 [26], that both express $M u c 2$ mRNA. The luciferase diagram shown in Fig. 2B, indicates that Muc2 promoter activity is the strongest in the CMT-93 cell line (black bars). In both cell lines, the highest luciferase activity was obtained with fragment $-729 /+29$. Since the activity increased from the fragment $-563 /+29$ to $-729 /+29$, it indicates that the $-729 /-564$ region possesses essential positive regulatory elements that confer maximal activity to the promoter. In both cell lines, a decreased activity was seen with fragment $-1568 /+29$ when compared with fragments $-1001 /+29$ and $-2213 /+29$. This indicates that there are inhibitory elements present within the $-1568 /-1002$ region of the promoter. In conclusion, despite being active in both cell lines, Muc2 promoter appears more active in goblet-like CMT-93 cells than in crypt-like IEC-6 cells.

GATA-4 regulates Muc2 expression at the transcriptional level

Effect of GATA-4 overexpression on Muc2 endogenous expression in CMT-93 cells was studied by RTPCR (Fig. 3A). The result indicates that $M u c 2$ mRNA level is substantially increased when GATA-4 is overexpressed in the cells (lane 2) when compared with cells transfected with the empty vector (lane 1 ). In order to identify GATA-4 responsive regions within $M u c 2$ promoter, co-transfection experiments were then carried out in the presence of pGL3-Muc2 deletion mutant $-221 /+29$ and GATA-4 expression vector (Fig. 3B, black bars). The luciferase diagram indicates that overexpression of GATA-4 resulted in a 10-fold increase of luciferase activity of the $-221 /+29$ construct. When a 

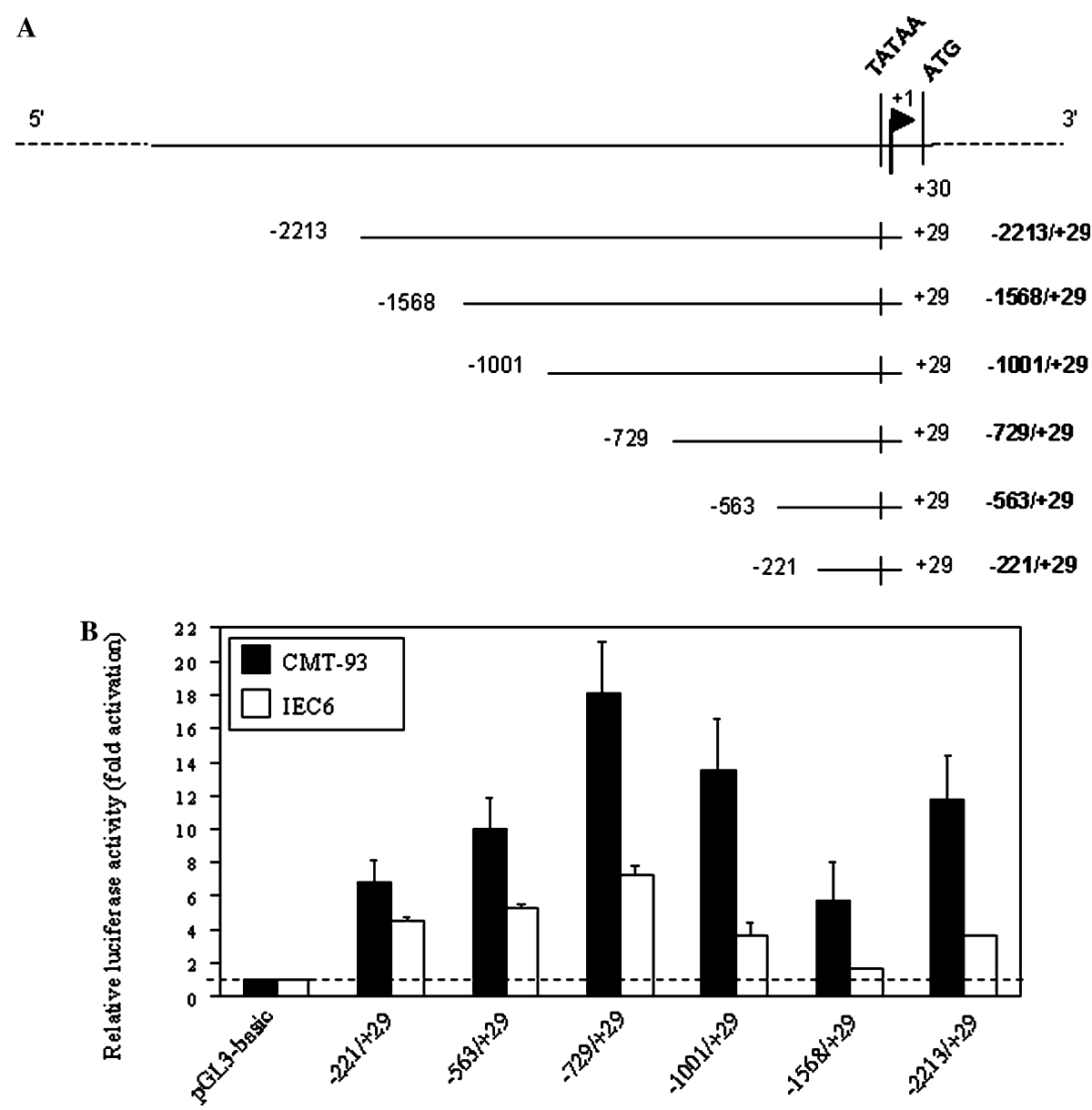

Fig. 2. Muc2 promoter constructs and promoter activity in CMT-93 and IEC-6 intestinal cells. (A) Schematic representation of the different deletion mutants used to study $\mathrm{Muc2}$ promoter activity. Numbering refers to transcription initiation site designated +1 . (B) Luciferase activity diagrams showing Muc2 promoter activity in murine rectal CMT-93 (black bars) and rat colon IEC-6 cells (white bars). Results are expressed as fold activity of the deletion mutant of interest compared with the activity of empty pGL3 Basic vector. Results represent means \pm SD obtained in triplicate in three separate experiments.

mutated form of the $-221 /+29$ construct was used, in

which the GATA sites at $-168 /-165$ and $-158 /-155$ were mutated (GATA to CTTA, see Table 2), $30 \%$ of the transactivating activity was lost (white bars). The fact that the luciferase activity did not return to basal levels suggests that either other GATA sites are present with the $-221 /+29$ region and contribute to the GATA4-mediated transactivating activity or that GATA-4 acts indirectly on the promoter via interactions with co-factors. In order to investigate the latter hypothesis, we co-transfected cells in the presence of the $-221 /+29$ deletion construct with either wild-type GATA-4 vector or mutated forms (-Act and C290S) (Fig. 3C). The -Act GATA-4 vector encodes a mutated form of GATA-4 in which the activation domains were deleted whereas C290S mutant encodes a GATA-4 form bearing a point mutation at amino acid position 290 (cysteine to serine) in the $\mathrm{COOH}$-terminal zinc finger [13]. Both forms are not able to bind DNA cognate element. Cotransfections with the wild-type vector (WT GATA-4) led to a twofold activation of both fragments tested $(-221 /+29$ and $-563 /+29)$. When the same experiment was performed with the mutated GATA-4 vectors (-Act and C290S), the luciferase activity decreased and returned to basal levels. This implicates that the transcription activation of the $M u c 2$ promoter by GATA-4 is mediated by a direct interaction of GATA4 with the DNA sequence. In conclusion, these experiments demonstrate that (i) GATA-4 induces $M u c 2$ transcription, (ii) responsive elements are present within the $-221 /+29$ region of the promoter, and (iii) GATA-4 binds directly to the promoter to transactivate $\mathrm{Muc2}$ promoter.

Identification of GATA-4 cis-elements within Muc2 promoter

Analysis of the proximal part of $M u c 2$ promoter sequence with MatInspector V2.2 and Alibaba2 software indicated the presence of four several putative GATA 

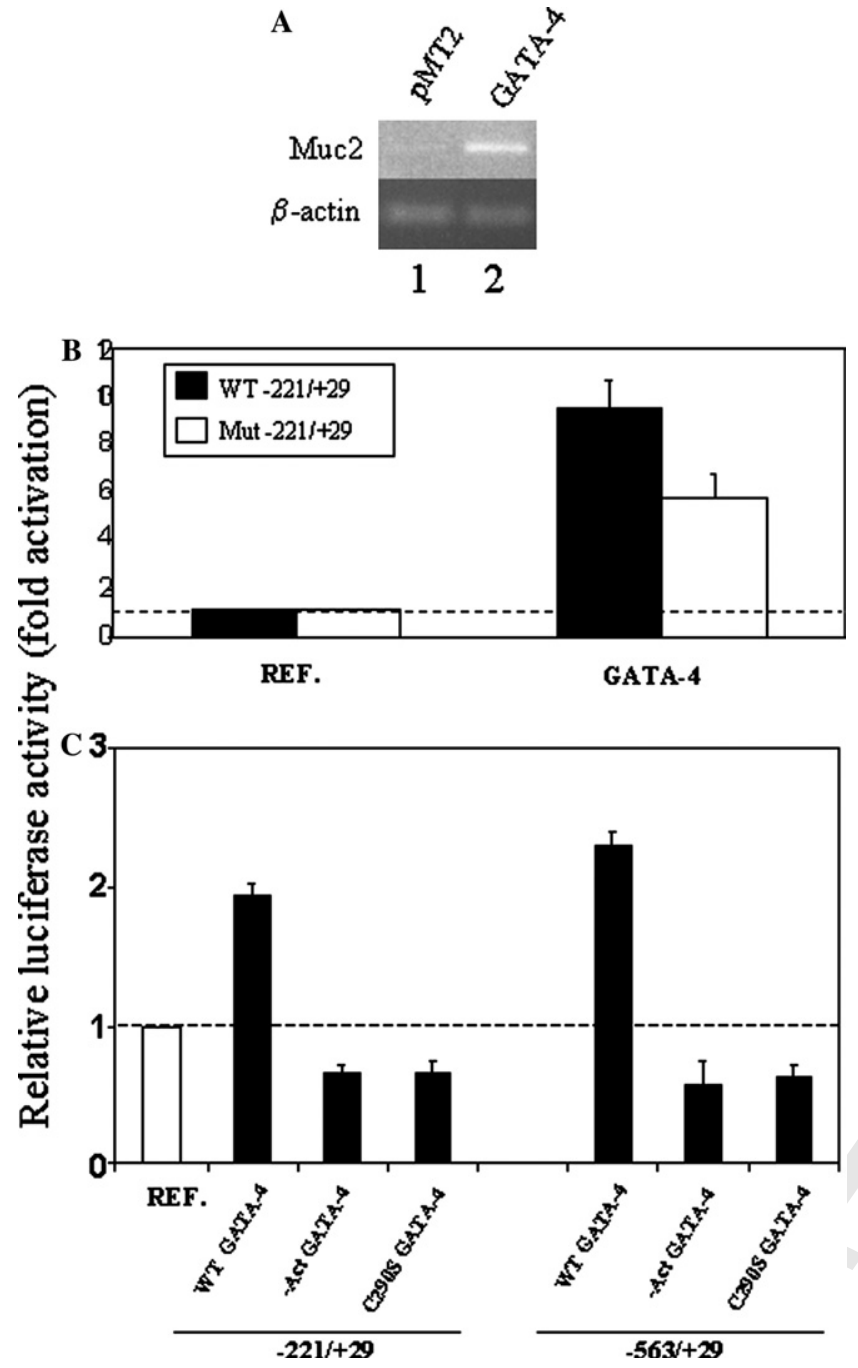

Fig. 3. Activation of $M u c 2$ mRNA level and promoter activity by GATA-4 in CMT-93 cells by RT-PCR and transfection assays, respectively. (A) Endogenous expression of $M u c 2$ mRNA after cotransfection with GATA-4 expression vector (lane 2) or corresponding empty vector (lane 1). $10 \mu \mathrm{l}(\mathrm{Muc2})$ and $2 \mu \mathrm{l}$ ( $\beta$-actin) of the PCR products were loaded on a $1.5 \%$ agarose gel and electrophoresed in a $1 \times$ Tris-borate buffer in the presence of ethidium bromide. (B) Luciferase diagram showing the effects of overexpression of GATA-4 on wild-type deletion construct $-221 /+29$ (black bars) or deletion construct $-221 /+29$ with two mutated GATA-4 sites at $-168 /-165$; $-158 /-155$ (white bars). (C) Luciferase diagram showing the effects of co-transfection of wild-type $-221 /+29$ or $-563 /+29$ pGL3-Muc 2 deletion mutants with wild-type GATA-4 (wt GATA-4) or mutated GATA-4 expression vectors (-Act GATA-4 and C290S GATA-4). Results are expressed as fold activation of luciferase activity in samples co-transfected with the transcription factor of interest compared with the control co-transfected with the corresponding empty vector (REF). Results represent means \pm SD obtained in triplicate in three separate experiments.

327 binding sites at $-99 /-96,-168 /-165,-158 /-155$, and $328-401 /-398$, respectively (Fig. 4A). They all contain the 329 conserved consensus sequence required for GATA bind330 ing. To confirm the binding of GATA-4 to these sites, 331 EMSAs were carried out in the presence of nuclear extracts prepared from CMT-93 cells. As shown in Figs. 4B and C three GATA-4 binding sites were identified in the T216 (one GATA site at -99/-96) and T168 (two GATA sites at $-168 /-165$ and $-158 /-155)$ probes, respectively. When radiolabeled probes were incubated with nuclear extracts, two specific shifted bands were visualized with T216 probe (Fig. 4B, lane 2) whereas only one was seen with T168 probe (Fig. 4C, lane 2). Specificity of the protein-DNA complexes was confirmed by the loss of the bands when cold competitions were performed with a $50 \times$ excess of the cold probes (Figs. 4B and C, lanes 3). As expected, cold competition with a $50 \times$ excess of a cold T168 mutated probe (Fig. $4 \mathrm{C}$, lane 4) did not modify the pattern of the shift. Moreover, when the mutated T168 probe was radiolabeled and incubated with nuclear extracts, no shift corresponding to the GATA binding was visualized (Fig. $4 \mathrm{C}$, lane 7). The same result was obtained with radiolabeled mutated T216 (not shown). Finally, implication of GATA- 4 in the binding to the T216 and T168 probes was proven by the supershifts (ssGATA-4) obtained when nuclear extracts and radiolabeled probes were incubated with an anti-GATA-4 antibody (Figs. 4B, lane 4 and $4 \mathrm{C}$, lane 5 , respectively). The identification of another GATA-4 cis-element at -99/-96 may thus explain the result shown in Fig. 3B in which GATA-4 transactivation was not completely lost when GATA sites at $-168 /-165$ and $-158 /-155$ were mutated. That third proximal GATA-4 cis-element is therefore most likely involved in the regulation of proximal Muc2 promoter by GATA-4. Of interest, this element (-99/-96) is conserved in the promoter of human MUC2 [24,27], which makes it central in the regulation of $M U C 2$ by GATA-4 between species. When EMSA was performed with radiolabeled probe T225 containing the fourth putative GATA binding site at $-401 /-398$, no binding was observed, indicating that no GATA factor is binding to that site (not shown).

Having found that GATA-4 transactivates the $2.2 \mathrm{~kb}$ fragment of $M u c 2$ promoter (not shown) and that a putative GATA binding site was present at -1521 / -1518 , we undertook to study whether GATA-4 was binding to that site. The result is shown in Fig. 4D. As for T168, when radiolabeled T169 was incubated with CMT-93 nuclear extracts one specific retarded band was visualized (GATA, lane 10). The binding was lost when cold competition was carried out in the presence of a $50 \times$ excess of the cold T169 probe (lane 11) whereas nothing happened when cold mutated probe was used in the competition (lane 12). Involvement of the GATA consensus sequence in the binding was confirmed by absence of complex formation when the mutated probe was radiolabeled and incubated with nuclear extracts (lane 15). Finally, binding of GATA-4 on that element was confirmed when supershift analysis was performed in the presence of an anti-GATA-4 antibody in the reac- 
A

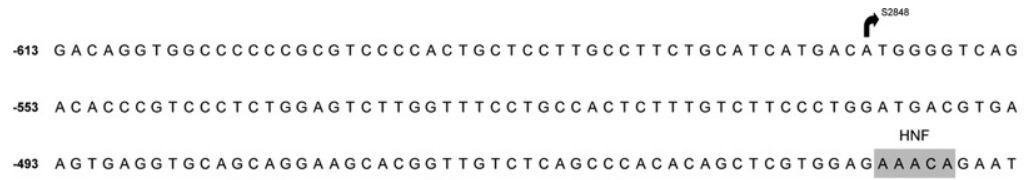

Nkx2.5
.433 GGAGTCGGGGGCACTTGAAAGC T225 TCTTATTCTATCTAGGCTGGGCTGGAGGTTGTGGGG

Nkx2.5
-433 GGAGTCGGGGACACTTGAAAGC T225 TCCTTATTCTATCTAGGCTGGGCTGGAGGTTGTGGG

Nkx2.5
-433 GGAGTCGGGGACACTTGAAAGC T225 TCCTTATTCTATCTAGGCTGGGCTGGAGGTTGTGGG

.373 CTGGGCCTGAGAGCATTGAGAAGGGTAGGCAGACCTTGTGGGATGTAATTCCAGCAGGC

-313 ACCTCCTGCCTTTCCCAACCTGAGTCTGGCCTGGCTTGTAGCTTAAAGGAATGGAGTTAG 253 TTCACCTGGGGTGTGCTGGCATTGGGaATTGG

250 TTCACCTGGGGTGTGTGCTGGCATTGGGATAATTGGGGCTATGACATCCTGAGGAAGCC 193 AAGTTTACCCAGGGAG TCATATAAAGATAAACTCAGATAACCTGAATCAATATTTCCTC -133 CTGGGACCCATGGAGCCCC CACAGCTGTTTTCCTGATAACTTGGCAAATGCCCCACCCAC -73 CCTTCGCACCTCCCTCGTCCTCCGCCCTCGGATGCCTGCTAAGCATATAAGTCCTGGACC 3 CCTGCCCTGGGATGCTCTOTO

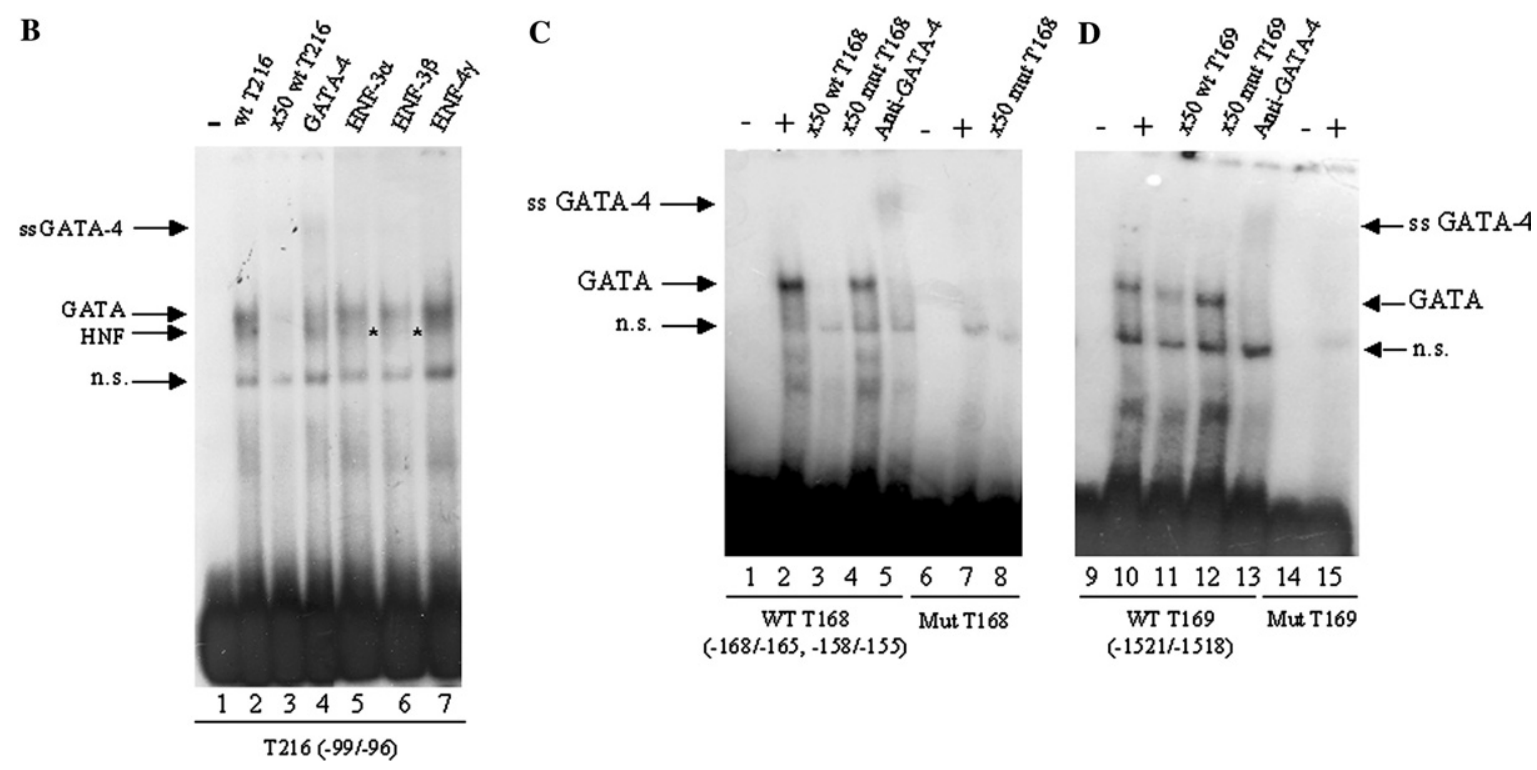

Fig. 4. Sequence of the proximal part of $M u c 2$ promoter and identification of GATA-4 cis-elements by EMSA. (A) The transcription site +1 (bold and underlined) is located 29 nucleotides downstream of the TATA box. Gray boxes indicate putative binding sites for transcription factors and boxed sequences indicate the sequences of oligonucleotides used in gel-shift assays. Arrows delineate the sequence of the deletion mutants used in this study. (B) Nuclear extracts were incubated with radiolabeled probe T216 (lanes 1-7). Probe alone (lane 1), radiolabeled T216 with nuclear extract (lane 2), and cold competition with 50x excess of wt T216 probe (lane 3). Supershift analysis was performed by preincubating the nuclear extract with $1 \mu \mathrm{l}$ of anti-GATA-4 (lane 4), anti-HNF-3 $\alpha$ (lane 5), anti-HNF-3 $\beta$ (lane 6), or anti-HNF-4 $\gamma$ (lane 7) antibodies, respectively. (C) Nuclear extracts were incubated with wild-type (lanes 1-5) and mutated (lanes 6-8) radiolabeled T168 probe, respectively. Probe alone (lanes 1 and 6), radiolabeled

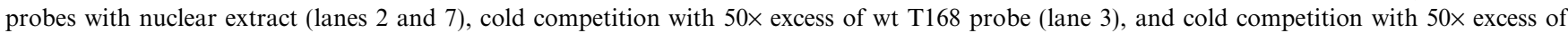
mutated T168 probe (lanes 4 and 8). Supershift analysis with $1 \mu$ anti-GATA-4 antibody (lane 5). (D) Nuclear extracts were incubated with wild-type (lanes 9-13) and mutated (lanes 14 and15) radiolabeled T169 probe, respectively. Probe alone (lanes 9 and 14), radiolabeled probes with nuclear extract (lanes 10 and 15), cold competition with 50× excess of wt T169 probe (lane 11), and cold competition with $50 \times$ excess of mutated T169 probe (lane 12). Supershift analysis with $1 \mu \mathrm{l}$ anti-GATA-4 antibody (lane 13).

388 tion mixture and resulted in a complete supershifting of

389 the protein-DNA complex (ssGATA-4, lane 13).

390 Of interest, we noticed that when the radioactive 391 probe T216 was incubated with nuclear extracts (Fig. 392 4B, lane 2) two specific shifted bands were visualized. 393 As described above, the low mobility complex corre394 sponds to a GATA-4 cis-element. Analysis of the se395 quence showed that the T216 probe also contained a 396 putative HNF-3 binding site (see Fig. 4A), that may 397 be responsible for the second shifted band. To confirm implication of HNF-3 factors in the binding, we performed supershift analysis with specific anti-HNF-3 $\alpha$ and anti-HNF-3 $\beta$ antibodies, and one irrelevant antiHNF- $4 \gamma$ antibody, respectively. As can be seen in Fig. $4 \mathrm{~B}$, incubation with anti-HNF-3 $\alpha$ (lane 5) and antiHNF-3 $\beta$ (lane 6) resulted in the disappearance of the high mobility complex (HNF), indicated by an asterisk, whereas nothing happened with anti-HNF-4 $\gamma$ (lane 7) antibody. This result indicates that HNF-3 $\alpha$ and HNF-3 $\beta$ factors bind to their cognate element at 
$408-108 /-104$, located next to the GATA-4 cis-element at 409 -99/-96 within $M u c 2$ proximal promoter. Previous 410 studies have shown that transcription factors from $411 \mathrm{HNF}$ and GATA families are able to act synergistically 412 to transactivate target genes [28-30]. These mechanisms 413 imply either direct binding to DNA or physical interac414 tions between HNF and GATA factors. It is of impor415 tance as HNF3 factors are also involved in the 416 formation of mammalian gut endoderm, early lineage 417 specification, and intestinal cell differentiation [28,29]. 418 Preliminary results indicate that HNF-3 $\beta$ is also ex419 pressed in Muc2-expressing goblet cells in the murine 420 small intestine (I. Renes, unpublished data), which 421 makes it an important factor to consider in $\mathrm{Muc} 2 \mathrm{regu}-$ 422 lation as well. Cooperation between factors of the HNF 423 and GATA families has already been described such as 424 the interaction between GATA-5 and HNF-1 $\alpha$ to upre425 gulate LPH [14,31]. Our result suggests that such a 426 cooperation may exist between GATA-4 and HNF-3 $\alpha$ 427 or HNF-3 $\beta$ to regulate $M u c 2$. In conclusion, the binding 428 studies allowed us to identify three GATA-4 cis-ele429 ments within the proximal part of $M u c 2$ promoter at $430-99 /-96,-168 /-165$, and $-158 /-155$, respectively, 431 and one in its distal part at $-1521 /-1518$. Moreover, 432 a HNF-3 $\alpha / 3 \beta$ cis-element was identified at $-108 /-104$. 433 In conclusion, we have shown that GATA-4, which 434 co-localizes with Muc2 in intestinal goblet cells, is a 435 strong transactivator of $M u c 2$ gene expression. 436 GATA-4 induces $M u c 2$ transcription by directly binding 437 to its cognate cis-elements within the promoter. We also 438 showed that HNF-3 $\alpha$ and HNF-3 $\beta$ bind to a cis-element 439 within the proximal promoter. All these data are in fa440 vor of an important role for GATA-4 factor in Muc2 441 spatio-temporal expression pattern observed in embry442 onic, fetal, and adult small intestine, and identifies for 443 the first time $M u c 2$, a gene that is a marker of goblet 444 cells, as a direct target of transcription factors involved 445 in intestinal development and cell differentiation. The 446 identification of GATA-4 as a main regulator of $M u c 2$ 447 is not only important for intestine differentiation but 448 also in other tissues where both GATA-4 and MUC2 449 are co-expressed. The gastric epithelium in which 450 GATA-4 is also expressed [32] is a good example, espe451 cially in gastric cancers associated with development of 452 intestinal metaplasia which are characterized by the ec453 topic expression of Muc2 [33]. Thus, GATA-4 appears 454 as an important general regulator of $M u c 2$ expression 455 and identifies $M u c 2$ as a target gene of GATA-4 in dif456 ferentiated intestinal mucosa and metaplastic stomach.

\section{Acknowledgments}

458 We thank Dr. S. Cereghini (INSERM U423, Hôpital 459 Necker, Paris) for the kind gift of the pMT2-GATA-4 460 expression vector and Dr. S.D. Krasinski (Harvard Med- ical School, Boston, MA, USA) for the gift of pcDNA3 (WT)-GATA-4 vector and its mutated forms pcDNA3 (-Act)-GATA-4 and pcDNA3 (C290S)-GATA-4. We are grateful to Dr. D. Podolsky (Massachusetts General Hospital, Boston, MA, USA) for providing us with the murine rectal cancer cell line CMT-93. This work was supported by grants from the Sophia Foundation for Medical Research (M.V.D.S. and A.W.C.E.), from INSERM/ ZonMw-NWO (I.V.S. and A.W.C.E.), from l'Association de Recherche sur le Cancer (No. 5785, I.V.S.), from la Ligue Régionale du Pas de Calais Contre le Cancer (I.V.S.), from l'Association François Aupetit (I.V.S.), and from the European Regional Development Fund. Nicolas Jonckheere is the recipient of a Conseil Régional Nord-Pas de Calais and Centre Hospitalier and Universitaire de Lille Ph.D. fellowship.

\section{References}

[1] A.P. Corfield, D. Carroll, N. Myerscough, C.S. Probert, Mucins in the gastrointestinal tract in health and disease, Front. Biosci. 6 (2001) D1321-D1357.

[2] K.M. Tytgat, H.A. Buller, F.J. Opdam, Y.S. Kim, A.W. Einerhand, J. Dekker, Biosynthesis of human colonic mucin: Muc2 is the prominent secretory mucin, Gastroenterology 107 (1994) 1352-1363.

[3] B.J. van Klinken, A.W. Einerhand, L.A. Duits, M.K. Makkink, K.M. Tytgat, I.B. Renes, M. Verburg, H.A. Buller, J. Dekker, Gastrointestinal expression and partial cDNA cloning of murine Muc2, Am. J. Physiol. 276 (1999) G115-G124.

[4] M.P. Buisine, L. Devisme, T.C. Savidge, C. Gespach, B. Gosselin, N. Porchet, J.P. Aubert, Mucin gene expression in human embryonic and fetal intestine, Gut 43 (1998) 519-524.

[5] I. Van Seuningen, P. Pigny, M. Perrais, N. Porchet, J.P. Aubert, Transcriptional regulation of the $11 \mathrm{p} 15$ mucin genes. Towards new biological tools in human therapy, in inflammatory diseases and cancer? Front. Biosci. 6 (2001) D1216-D1234.

[6] P. Mesquita, N. Jonckheere, R. Almeida, M.P. Ducourouble, J. Serpa, E. Silva, P. Pigny, F.S. Silva, C. Reis, D. Silberg, I. Van Seuningen, L. David, Human MUC2 mucin gene is transcriptionally regulated by $\mathrm{Cdx}$ homeodomain proteins in gastrointestinal carcinoma cell lines, J. Biol. Chem. 278 (2003) 51549-51556.

[7] D.G. Silberg, G.P. Swain, E.R. Suh, P.G. Traber, Cdx1 and cdx2 expression during intestinal development, Gastroenterology 119 (2000) 961-971.

[8] J.D. Molkentin, The zinc finger-containing transcription factors GATA-4, -5, and -6. Ubiquitously expressed regulators of tissuespecific gene expression, J. Biol. Chem. 275 (2000) 38949-38952.

[9] E.E. Morrisey, H.S. Ip, M.M. Lu, M.S. Parmacek, GATA-6: a zinc finger transcription factor that is expressed in multiple cell lineages derived from lateral mesoderm, Dev. Biol. 177 (1996) 309-322.

[10] E.E. Morrisey, H.S. Ip, Z. Tang, M.M. Lu, M.S. Parmacek, GATA-5: a transcriptional activator expressed in a novel temporally and spatially-restricted pattern during embryonic development, Dev. Biol. 183 (1997) 21-36.

[11] X. Gao, T. Sedgwick, Y.B. Shi, T. Evans, Distinct functions are implicated for the GATA-4, -5 , and -6 transcription factors in the regulation of intestine epithelial cell differentiation, Mol. Cell. Biol. 18 (1998) 2901-2911.

[12] R. Fang, L.C. Olds, N.A. Santiago, E. Sibley, GATA family transcription factors activate lactase gene promoter in intestinal 
Caco-2 cells, Am. J. Physiol. Gastrointest. Liver Physiol. 280 (2001) G58-G67.

[13] H.M. Van Wering, T. Bosse, A. Musters, E. De Jong, N. De Jong, C.E. Hogen Esch, F. Boudreau, G.P. Swain, L.N. Dowling, R.K. Montgomery, R.J. Grand, S.D. Krasinski, Complex regulation of the lactase-phlorizin hydrolase promoter by GATA-4, Am. J. Physiol. Gastrointest. Liver Physiol. 287 (2004) G899G909.

[14] S.D. Krasinski, H.M. Van Wering, M.R. Tannemaat, R.J. Grand, Differential activation of intestinal gene promoters: functional interactions between GATA-5 and HNF-1 alpha, Am. J. Physiol. Gastrointest. Liver Physiol. 281 (2001) G69-G84.

[15] F. Aslam, L. Palumbo, L.H. Augenlicht, A. Velcich, The Sp family of transcription factors in the regulation of the human and mouse MUC2 gene promoters, Cancer Res. 61 (2001) 570-576.

[16] I.B. Renes, J.A. Boshuizen, D.J. Van Nispen, N.P. Bulsing, H.A. Buller, J. Dekker, A.W. Einerhand, Alterations in Muc2 biosynthesis and secretion during dextran sulfate sodium-induced colitis, Am. J. Physiol. Gastrointest. Liver Physiol. 282 (2002) G382G389.

[17] I. Van Seuningen, M. Perrais, P. Pigny, N. Porchet, J.P. Aubert, Sequence of the $5^{\prime}$-flanking region and promoter activity of the human mucin gene MUC5B in different phenotypes of colon cancer cells, Biochem. J. 348 (Pt. 3) (2000) 675-686.

[18] M. Perrais, P. Pigny, M.C. Copin, J.P. Aubert, I. Van Seuningen, Induction of MUC2 and MUC5AC mucins by factors of the epidermal growth factor (EGF) family is mediated by EGF receptor/Ras/Raf/extracellular signal-regulated kinase cascade and Sp1, J. Biol. Chem. 277 (2002) 32258-32267.

[19] N. Jonckheere, M. Van Der Sluis, A. Velghe, M.P. Buisine, M. Sutmuller, M.P. Ducourouble, P. Pigny, H.A. Buller, J.P. Aubert, A.W. Einerhand, I. Van Seuningen, Transcriptional activation of the murine Muc5ac mucin gene in epithelial cancer cells by TGFbeta/Smad4 signalling pathway is potentiated by Sp1, Biochem. J. 377 (2004) 797-808.

[20] I. Van Seuningen, J. Ostrowski, X.R. Bustelo, P.R. Sleath, K. Bomsztyk, The $\mathrm{K}$ protein domain that recruits the interleukin 1responsive $\mathrm{K}$ protein kinase lies adjacent to a cluster of $\mathrm{c}-\mathrm{Src}$ and Vav SH3-binding sites. Implications that $\mathrm{K}$ protein acts as a docking platform, J. Biol. Chem. 270 (1995) 26976-26985.

[21] K. Quandt, K. Frech, H. Karas, E. Wingender, T. Werner, MatInd and MatInspector: new fast and versatile tools for detection of consensus matches in nucleotide sequence data, Nucleic Acids Res. 23 (1995) 4878-4884.
[22] J.P. Audié, A. Janin, N. Porchet, M.C. Copin, B. Gosselin, J.P. Aubert, Expression of human mucin genes in respiratory, digestive, and reproductive tracts ascertained by in situ hybridization, J. Histochem. Cytochem. 41 (1993) 1479-1485.

[23] P.G. Traber, Epithelial cell growth and differentiation. V. Transcriptional regulation, development, and neoplasia of the intestinal epithelium, Am. J. Physiol. 273 (1997) G979-G981.

[24] A. Velcich, L. Palumbo, L. Selleri, G. Evans, L. Augenlicht, Organization and regulatory aspects of the human intestinal mucin gene (MUC2) locus, J. Biol. Chem. 272 (1997) 7968-7976.

[25] L.M. Franks, V.J. Hemmings, A cell line from an induced carcinoma of mouse rectum, J. Pathol. 124 (1978) 35-38.

[26] A. Quaroni, J. Wands, R.L. Trelstad, K.J. Isselbacher, Epithelioid cell cultures from rat small intestine. Characterization by morphologic and immunologic criteria, J. Cell Biol. 80 (1979) 248265.

[27] J.R. Gum, J.W. Hicks, Y.S. Kim, Identification and characterization of the MUC2 (human intestinal mucin) gene $5^{\prime}$-flanking region: promoter activity in cultured cells, Biochem. J. 325 (Pt. 1) (1997) 259-267.

[28] K. Zaret, Developmental competence of the gut endoderm: genetic potentiation by GATA and HNF3/fork head proteins, Dev. Biol. 209 (1999) 1-10.

[29] P.G. Traber, D.G. Silberg, Intestine-specific gene transcription, Annu. Rev. Physiol. 58 (1996) 275-297.

[30] F. Boudreau, E.H. Rings, H.M. van Wering, R.K. Kim, G.P. Swain, S.D. Krasinski, J. Moffett, R.J. Grand, E.R. Suh, P.G. Traber, Hepatocyte nuclear factor-1 alpha, GATA-4, and caudal related homeodomain protein $\mathrm{Cdx} 2$ interact functionally to modulate intestinal gene transcription. Implication for the developmental regulation of the sucrase-isomaltase gene, J. Biol. Chem. 277 (2002) 31909-31917.

[31] H.M. van Wering, I.L. Huibregtse, S.M. vander Zwan, M.S. de Bie, L.N. Dowling, F. Boudreau, E.H. Rings, R.J. Grand, S.D. Krasinski, Physical interaction between GATA-5 and hepatocyte nuclear factor-1alpha results in synergistic activation of the human lactase-phlorizin hydrolase promoter, J. Biol. Chem. 277 (2002) 27659-27667.

[32] S. Tamura, X.H. Wang, M. Maeda, M. Futai, Gastric DNAbinding proteins recognize upstream sequence motifs of parietal cell-specific genes, Proc. Natl. Acad. Sci. USA 90 (1993) 1087610880.

[33] Y. Yuasa, Control of gut differentiation and intestinal-type gastric carcinogenesis, Nat. Rev. Cancer 3 (2003) 592-600. 